\title{
CHARACTERIZATION OF THE NEARBY L/T BINARY BROWN DWARF WISE J104915.57-531906.1 AT 2 pc FROM THE SUN*
}

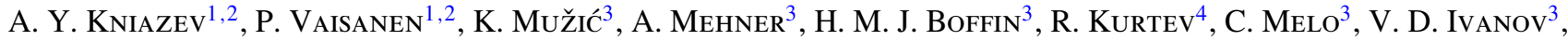 \\ J. Girard ${ }^{3}$, D. Mawet ${ }^{3}$, L. Schmidtobreick ${ }^{3}$, N. Huelamo ${ }^{5}$, J. Borissova ${ }^{4}$, D. Minniti ${ }^{6,7}$, K. Ishibashi ${ }^{8}$, \\ S. B. Potter ${ }^{1}$, Y. Beletsky ${ }^{9}$, D. A. H. Buckley ${ }^{2}$, S. Crawford ${ }^{1,2}$, A. A. S. Gulbis ${ }^{1,2}$, P. Kotze ${ }^{1,2}$, \\ B. Miszalski ${ }^{1,2}$, T. E. Pickering ${ }^{1,2}$, E. Romero Colmenero ${ }^{1,2}$, AND T. B. Williams ${ }^{1,10,11}$ \\ ${ }^{1}$ South African Astronomical Observatory, P.O. Box 9, 7935 Observatory, Cape Town, South Africa \\ ${ }^{2}$ Southern African Large Telescope Foundation, P.O. Box 9, 7935 Observatory, Cape Town, South Africa \\ ${ }^{3}$ European Southern Observatory, Ave. Alonso de Cordova 3107, Casilla 19001, Santiago 19, Chile \\ ${ }^{4}$ Departamento de Física y Astronomía, Universidad de Valparaiso, Av. Gran Bretaña 1111, Playa Ancha, 5030 Casilla, Chile \\ ${ }^{5}$ CAB (INTA-CSIC), LAEFF, P.O. Box 78, E-28691 Villanueva de la Cañada, Madrid, Spain \\ ${ }^{6}$ Departamento Astronomía y Astrofísica, Pontificia Universidad Católica de Chile, Av. Vicuña Mackenna 4860, Santiago, Chile \\ ${ }^{7}$ Vatican Observatory, V-00120 Vatican City State, Italy \\ ${ }^{8}$ Nagoya University, Nagoya, Japan \\ ${ }^{9}$ Las Campanas Observatory, Carnegie Institution of Washington, Colina el Pino, Casilla 601 La Serena, Chile \\ ${ }^{10}$ Department of Astronomy, University of Cape Town, Cape Town, South Africa \\ ${ }^{11}$ Department of Physics and Astronomy, Rutgers, The State University of New Jersey, Piscataway, NJ 08854, USA \\ Received 2013 March 28; accepted 2013 May 2; published 2013 June 4
}

\begin{abstract}
WISE J104915.57-531906.1 is a L/T brown dwarf binary located 2 pc from the Sun. The pair contains the closest known brown dwarfs and is the third closest known system, stellar or sub-stellar. We report comprehensive follow-up observations of this newly uncovered system. We have determined the spectral types of both components (L8 \pm 1 , for the primary, agreeing with the discovery paper; T1.5 \pm 2 for the secondary, which was lacking spectroscopic type determination in the discovery paper) and, for the first time, their radial velocities $\left(V_{\mathrm{rad}} \sim 23.1\right.$, $19.5 \mathrm{~km} \mathrm{~s}^{-1}$ ) using optical spectra obtained at the Southern African Large Telescope and other facilities located at the South African Astronomical Observatory (SAAO). The relative radial velocity of the two components is smaller than the range of orbital velocities for theoretically predicted masses, implying that they form a gravitationally bound system. We report resolved near-infrared $J H K_{S}$ photometry from the Infrared Survey Facility telescope at the SAAO which yields colors consistent with the spectroscopically derived spectral types. The available kinematic and photometric information excludes the possibility that the object belongs to any of the known nearby young moving groups or associations. Simultaneous optical polarimetry observations taken at the SAAO $1.9 \mathrm{~m}$ give a non-detection with an upper limit of $0.07 \%$. For the given spectral types and absolute magnitudes, 1 Gyr theoretical models predict masses of $0.04-0.05 M_{\odot}$ for the primary, and 0.03-0.05 $M_{\odot}$ for the secondary.
\end{abstract}

Key words: brown dwarfs - infrared: stars - solar neighborhood - stars: individual (G 171-22, HD 55383, WISE J104915.57-531906.1) - stars: low-mass

Online-only material: color figures

\section{INTRODUCTION}

Nearby stars are easy to identify from their high proper motion (PM) which can reach many tenths of arcseconds per year. However, the confusion against the crowded Milky Way background can make such objects hard to identify near the Galactic plane. The extreme red colors of late-type objects make their detection by the optical surveys challenging. The last few decades have seen great improvements with both these issues. A number of projects have generated rich data sets for PM searches in the near-infrared (NIR) and mid-infrared: Two Micron All Sky Survey (2MASS; Skrutskie et al. 2006), the Deep NearInfrared Survey of the Southern Sky (DENIS; Epchtein et al. 1999), and the United Kingdom Infrared Telescope Infrared Deep Sky Survey (UKIDSS; Lawrence et al. 2007). Most recently, the Wide-field Infrared Survey Explorer (WISE; Wright et al. 2010) imaged the entire sky at 3.4, 4.6, 12, and $22 \mu \mathrm{m}$.

\footnotetext{
* Based on observations made with the Southern African Large Telescope (SALT).
}

This mission is particularly sensitive to sub-stellar objects, and it obtained observations over many epochs separated by $0.5-1 \mathrm{yr}$.

Luhman (2013) used these multi-epoch WISE observations to search for objects with red colors and high PM. He identified WISE J104915.57-531906.1 (hereafter W10-53) with $\mu \sim$ $3^{\prime \prime} \mathrm{yr}^{-1}$. Follow-up observations showed two objects at the location of W10-53 and spectroscopy of the primary indicated an L8 spectral type. A parallax based on WISE and a number of older surveys placed W10-53 at a distance of $\sim 2 \mathrm{pc}$. This makes it the third closest system to the Sun, after Proxima/ $\alpha$ Cen and Barnard's star (Barnard 1916). These are now the closest brown dwarfs (BDs), usurping UGPS 0722-05 (Lucas et al. 2010) from this position. Additional archival detections were reported by Mamajek (2013). He used kinematic considerations to conclude that the system probably belongs to the thin disk and is unlikely to be younger than $10^{8} \mathrm{yr}$. Gandhi et al. (2013) reported X-ray non-detection setting an X-ray-to-bolometricluminosity limit of W10-53 to $\log \left(L_{0.2-3 \mathrm{kev}} / L_{\mathrm{bol}}\right)<-4.6$, consistent with this age.

W10 -53 , together with the other recently discovered nearby BDs (Lucas et al. 2010; Artigau et al. 2010), can constrain 


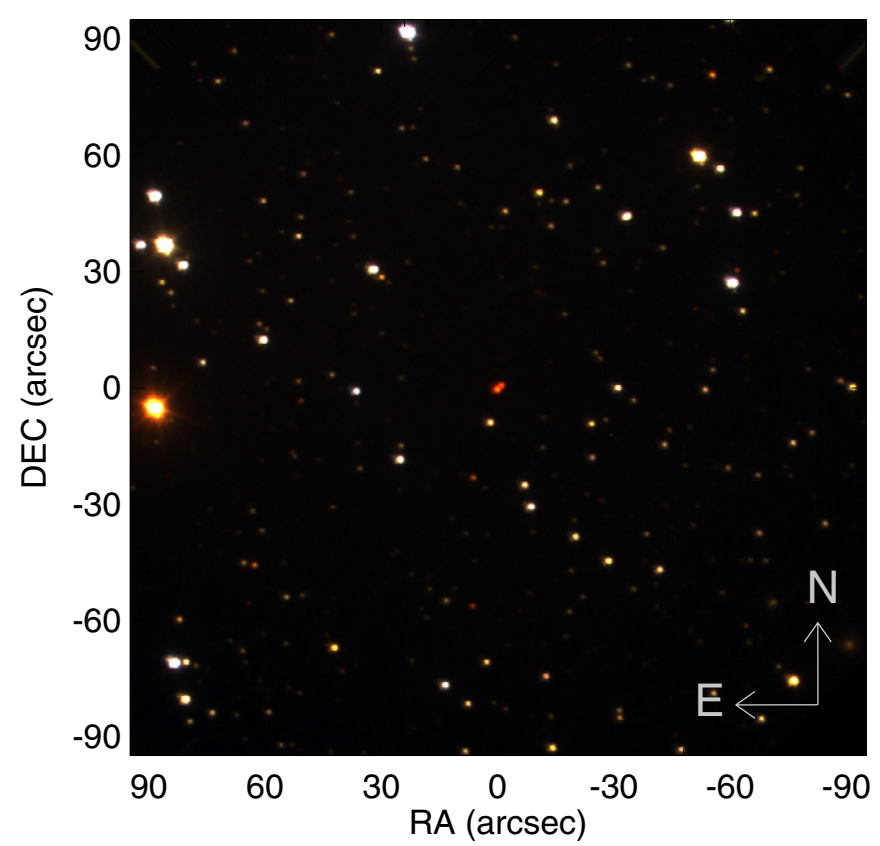

Figure 1. Three-color optical image of the W10-53 field highlighting the extremely red color of the binary. Three $90 \mathrm{~s}$ exposures taken with the RSS at SALT at R.A. $=10^{\mathrm{h}} 49^{\mathrm{m}} 15^{\mathrm{s}} .57$, decl. $=-53^{\circ} 19^{\prime} 06^{\prime \prime} .1$ were combined: red, green, and blue correspond to 100-200 Å wide filters centered at 8175, 7260, and $5060 \AA$, respectively.

(A color version of this figure is available in the online journal.)

the local BD density and offers us an opportunity to study these cool objects in detail, search for planets around them, and even resolve their surfaces with future interferometric instruments. We carried out optical spectroscopy, NIR imaging, and optical polarimetry of W10-53 with Southern African Large Telescope (SALT) and other facilities at the South African Astronomical Observatory (SAAO) to obtain spectral types of both components, measure their radial velocities, test if the system may belong to a nearby moving group or association, and look for the presence of scattering dust.

\section{OBSERVATIONS AND DATA REDUCTION}

\subsection{SALT Optical Spectroscopy}

Long-slit spectra of W10-53 were obtained with the Robert Stobie Spectrograph (RSS; Burgh et al. 2003; Kobulnicky et al. 2003) at the SALT (Buckley et al. 2006; O'Donoghue et al. 2006) in Sutherland, South Africa. The RSS uses a mosaic of three $2048 \times 4096$ CCDs. The spatial scale was $0^{\prime \prime} .253$ pixel $^{-1}$, after binning by a factor of two. The $0^{\prime \prime} 6$ wide slit was rotated to a position angle of $133^{\circ}$ to observe both objects simultaneously. SALT makes use of an Atmospheric Dispersion Compensator, ensuring that there were no colordependent slit losses. The PG1800 grating was used on 2013 March 12 to measure the radial velocities because it provides the highest spectral resolution at red wavelengths, resulting in spectral coverage of 7870-8940 $\AA$ and spectral resolution of $0.97 \AA(0.33 \AA$ per binned pixel $)$. To improve the spectral typing, a wider range $6700-9670 \AA$ was observed on 2013 March 16, with the PG900 grating, providing $2.19 \AA$ (1.89 $\AA$ per binned pixel) resolution. A single $600 \mathrm{~s}$ spectrum was taken in each set up. The seeing during both observations was 1".3-1".4 and the two components, separated by $\sim 1^{\prime \prime} .5$, were resolved on the acquisition images (Figure 1). A Neon lamp arc spectrum and a set of Quartz Tungsten Halogen (QTH) flats were taken immediately after the science frames. A spectrophotometric standard star, CD-32 9927 , was observed for both setups.

The overscan, gain, cross-talk corrections, and mosaicing were done using the SALT data pipeline, PySALT (Crawford et al. 2010). The red end ( $>8400 \AA)$ of the spectra suffer from significant fringing effects, but we were able to remove them using the QTH flats. MIDAS ${ }^{12}$ and routines from the twodspec package in IRAF $^{13}$ were used for wavelength calibration, frame rectification, and background subtraction of the twodimensional (2D) spectrum (Kniazev et al. 2008). The derived internal error for the wavelength calibration is $\sigma=0.03 \AA$ throughout the wavelength range. This was verified against the numerous night sky lines in this wavelength range. Velocities were then corrected for heliocentric motion. Absolute flux calibration is not feasible with SALT because the unfilled entrance pupil of the telescope moves during the observation. However, a relative flux correction to recover the spectral shape was done using the observed spectrophotometric standard.

The top panel of Figure 2 shows a section of the fully reduced PG1800 2D spectrum demonstrating that we were successful in spatially separating the binary components. The one-dimensional (1D) spectra were extracted with 5 pixel $\left(\sim 1^{\prime \prime} .3\right)$ apertures. We optimized the width and the location of the apertures to minimize the cross-contamination between the two companions: the spectrum of B contains less than $6 \%$ of the light from $\mathrm{A}$, and the spectrum of A contains less than $3 \%$ of the light from B. The lower panel of Figure 2 shows the wider wavelength range taken with the PG900 grating.

\subsection{IRSF NIR Imaging}

W10-53 was imaged with the Simultaneous-Color InfraRed Imager for Unbiased Survey (SIRIUS; Nagayama et al. 2003) on the Infrared Survey Facility (IRSF) $1.4 \mathrm{~m}$ telescope in Sutherland on 2013 March 16, under clear conditions and $\sim 0$ '.8 J-band seeing. SIRIUS has three $1024 \times 1024 \mathrm{HgCdTe}$ detectors and it splits the incoming light by two dichroics for simultaneous $J H K_{S}$ observations. The scale is 0.45 pixel $^{-1}$, yielding $\sim 7.7 \times 7.7$ arcmin field of view. We took a set of 10 dithered images with $5 \mathrm{~s}$ exposures for the individual frames. The data were reduced with the SIRIUS pipeline. ${ }^{14}$

The first reduction steps were flat-fielding and subtraction of dark current and sky background. Then, the 10 dithered frames were aligned and combined into a final image. The astrometric calibration was derived from 2MASS stars in the field. Forty-nine of the 2MASS stars, selected to have no close neighbors, were used to determine the flux zero points. These stars span wide color and magnitude ranges $\left(0.1 \mathrm{mag} \leqslant J-K_{\mathrm{S}} \leqslant 1.5 \mathrm{mag}, 9.6 \mathrm{mag} \leqslant K_{\mathrm{S}} \leqslant 15.6 \mathrm{mag}\right.$; but note that component $\mathrm{A}$ is somewhat outside the color range). The SIRIUS filter system is unique so we transferred the measured instrumental magnitudes into the 2MASS system. The color terms of our transformations are identical, within the uncertainties, to those of (Kuchinskas et al. 2008). A least-squares fit to the transformations yielded an absolute photometric uncertainty below $3 \%$.

\footnotetext{
12 Munich Image Data Analysis System is distributed by ESO.

13 IRAF is distributed by the National Optical Astronomy Observatory, which is operated by the Association of Universities for Research in Astronomy, Inc., under cooperative agreement with the National Science Foundation.

14 The pipeline can be retrieved at http://www.z.phys.nagoya-u.ac.jp/ $\sim$ nakajima/sirius/software/software.html.
} 

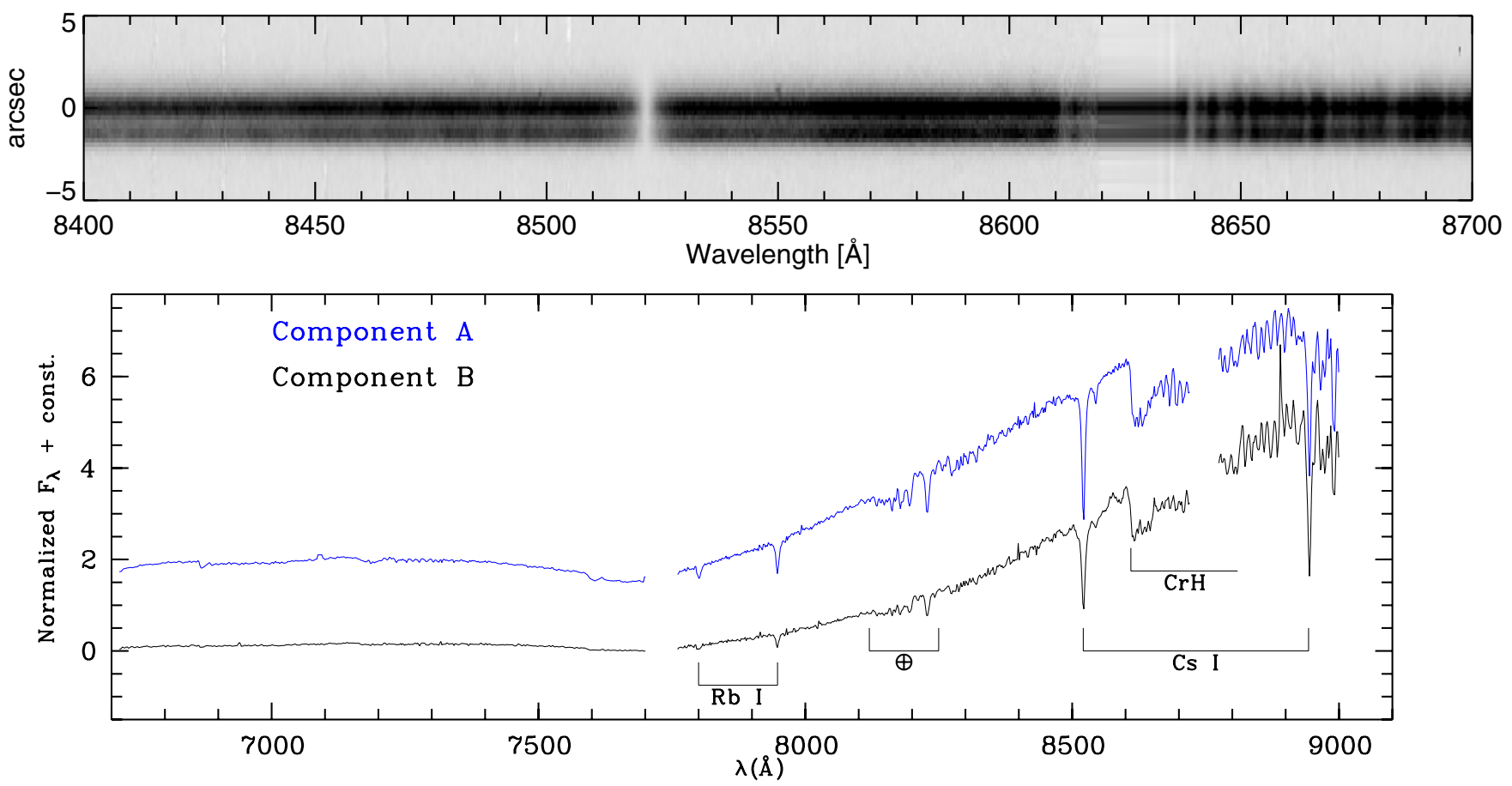

Figure 2. Top: part of the wavelength range of the reduced 2D spectrum of W10-53 taken with the PG1800 grating, showing the spatially resolved components of the binary. Component A, brighter in the optical, is at the top. The Cs I absorption line is clearly seen at $8521 \AA$, the CrH bandhead at $8610 \AA$ and the gap between RSS detectors at 8620-8635 ̊ are clearly seen. Bottom: the extracted 1D spectra of both components obtained with the PG900 grating. The main spectral features are marked.

(A color version of this figure is available in the online journal.)

Table 1

IRSF Photometry for W10-53 from 2013 March 16

\begin{tabular}{lrrr}
\hline \hline Band & Component $\mathrm{A}^{\mathrm{a}}$ & \multicolumn{1}{c}{${\text { Component } \mathrm{B}^{\mathrm{a}}}$} & \multicolumn{1}{c}{ Combined $^{\mathrm{a}}$} \\
\hline$J$ & $11.511 \pm 0.028$ & $11.233 \pm 0.028$ & $10.611 \pm 0.028$ \\
$H$ & $10.396 \pm 0.026$ & $10.369 \pm 0.028$ & $9.634 \pm 0.026$ \\
$K_{S}$ & $9.559 \pm 0.029$ & $9.767 \pm 0.029$ & $8.901 \pm 0.029$ \\
\hline
\end{tabular}

Note. ${ }^{\text {a }}$ Uncertainties represent the formal Poisson errors.

The stellar photometry was carried out with ALLSTAR in DAOPHOT II (Stetson 1987). The point spread function (PSF) width of $\sim 1^{\prime \prime} .0-11^{\prime \prime} .1$ and the $\sim 1^{\prime \prime} .5$ separation between the two components allowed us to achieve fitting errors of $\sim 0.008 \mathrm{mag}$. The apparent magnitudes of the two components are reported in Table 1. We list for comparison their combined magnitudes which are in excellent agreement with 2MASS and DENIS data.

\subsection{SAAO 1.9 m Optical Polarimetry}

W10-53 was observed with the HI-speed Photo-POlarimeter (HIPPO; Potter et al. 2010) on the $1.9 \mathrm{~m}$ telescope of the SAAO in simultaneous linear and circular polarimetry and photometry mode (all-Stokes) on 2013 March 19, under moonless photometric conditions. Measurements were performed with the Kron-Cousins $I$ filter for a duration of 40 minutes. Background sky measurements were acquired immediately prior to the observations. Polarized standards HD 298383 and HD 80558 were used to calculate the position angle offsets and efficiency factors. The two components of W10-53 were unresolved by the instrument. No polarization was detected with a firm upper limit of $0.07 \%$.

\section{ANALYSIS}

\subsection{Spectral Classification}

To determine the spectral types of the two components, we created a spectral sequence of field L- and T-dwarf primary spectral standards from Kirkpatrick et al. (1999) and Burgasser et al. (2003), adding extra L9 (Kirkpatrick et al. 2000), and T1 (classified in the NIR by McLean et al. 2003) templates. ${ }^{15}$ Figure 3 shows the PG900 spectra of W10-53 A and B (black) along with various spectral templates (purple for the primary and green for the secondary). Our spectra were smoothed by a boxcar function to a resolution of $\sim 3.3 \AA$ and normalized at $8200 \AA$.

The primary is best matched to L7-L9 spectra and we therefore classify it as L8 \pm 1 , the same as Luhman (2013). Typing of the secondary is less straightforward, mainly because of the lack of reliable spectral templates of T-dwarfs in the optical. The spectrum appears to be bracketed by T1 and T2, and we assign T1.5 \pm 2 to this object. Our resolved NIR photometry yields colors consistent with the colors of BDs with the same spectral types from the Dwarf Archives. ${ }^{16}$ Burgasser et al. (2013) presented resolved NIR spectroscopy of W10-53, deriving L7.5 \pm 0.9 and T0.5 \pm 0.7 , for the primary and secondary, respectively, consistent with our spectral types.

The measured apparent magnitudes combined with the astrometrically calibrated absolute magnitudes of Dupuy \& Liu (2012) yield distances for the primary and the secondary of $2.5 \pm 0.3$ and $2.0 \pm 0.2 \mathrm{pc}$, respectively. Their consistency with the parallax of Luhman (2013), together with the typical

\footnotetext{
$\overline{15}$ Data available at http://staff.gemini.edu/ $\sim$ sleggett/LTdata.html and http://www.iac.es/galeria/ege/catalogo_espectral

$16 \mathrm{http} / / /$ spider.ipac.caltech.edu/staff/davy/ARCHIVE/index.shtml
} 

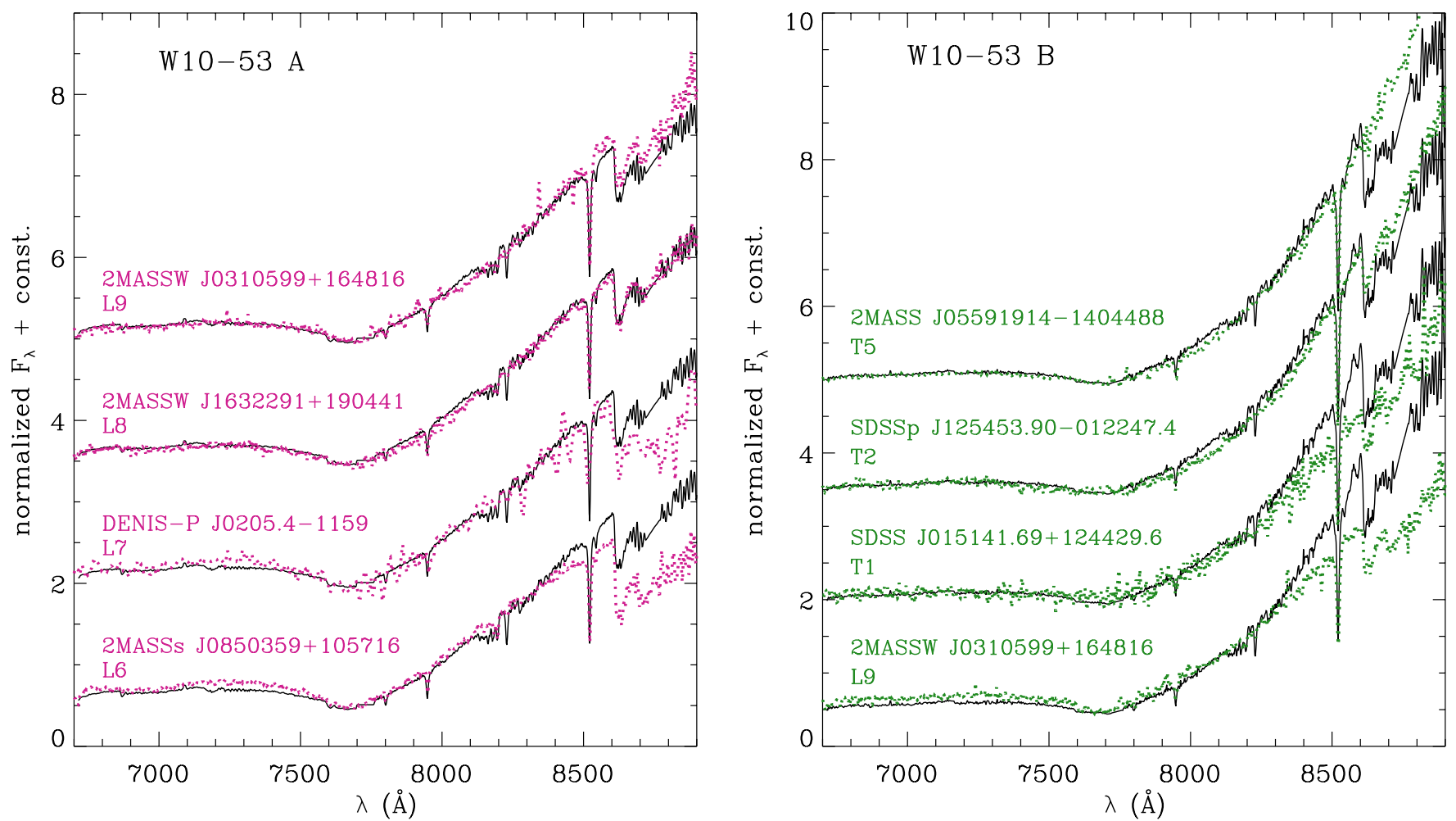

Figure 3. Comparison of the W10-53 A and B PG900 spectra (black) with various spectral templates (green and purple).

(A color version of this figure is available in the online journal.)

magnitudes of the components, make it unlikely to find that W10-53 is a nearly equal mass hierarchical BD system. However, the magnitudes must be treated with caution until further studies, because the photometric monitoring in $I+z$ by Gillon et al. (2013) yielded a quasi-periodic $(P=4.87 \pm 0.01 \mathrm{hr})$ variation of the secondary, probably produced by fast rotation and fast-changing cloud structure.

\subsection{Radial Velocity}

We estimated the radial velocities for both components from the SALT medium-resolution spectra. The two most prominent absorption lines were used, identified in both spectra as Rb I at 7947.60 $\AA$ and Cs I at $8521.13 \AA$ (Figure 2 and Kirkpatrick et al. 1999). To exclude systematic shifts originating from the known RSS flexure, we calculated the line-of-sight velocity distributions along the slit using the method and programs described in Zasov et al. (2000) where the nearest night-sky lines were used as references. The IRAF task splot was used to calculate the centers of the absorption lines providing the measured radial velocities $V_{\text {rad }}=23.1 \pm 1.1$ and $19.5 \pm 1.2 \mathrm{~km} \mathrm{~s}^{-1}$ for components $\mathrm{A}$ and $\mathrm{B}$, respectively. These values incorporate a heliocentric correction of $7.4 \mathrm{~km} \mathrm{~s}^{-1}$, corresponding to the observing time of 2013 March 13, 01:14 UT. We cross-correlated the SALT medium-resolution spectra of component A with component B to measure directly their relative velocity: $2.5 \pm 1.9 \mathrm{~km} \mathrm{~s}^{-1}$, consistent with the difference between the velocities given above: $3.6 \pm 1.6 \mathrm{~km} \mathrm{~s}^{-1}$.

As a double-check, we cross-correlated the spectra of the two components with a $1400 \mathrm{~K}$ synthetic spectrum computed with the Phoenix simulator, ${ }^{17}$ using the BT-Settl models (Allard et al. 2003). The cross-correlation, performed with the IRAF task xcsao, yielded $22.0 \pm 4.0 \mathrm{~km} \mathrm{~s}^{-1}$ and $18.2 \pm 4.2 \mathrm{~km} \mathrm{~s}^{-1}$,

\footnotetext{
17 http://phoenix.ens-lyon.fr/simulator/index.faces
}

in excellent agreement with the values obtained above. These estimates are less reliable because the template refers to averaged parameters of both components, and they use all lines, not just the strongest ones, subjecting the cross-correlation to more noise.

The observed PM of the star translates to a transverse velocity of $28.4 \mathrm{~km} \mathrm{~s}^{-1}$, and the total relative velocity of the star with respect to the Sun is $\sim 36 \mathrm{~km} \mathrm{~s}^{-1}$.

\subsection{Polarization}

Linear polarization in cool sub-stellar objects is thought to arise in disks, or due to dust in their atmospheres, combined with asymmetries caused by oblateness due to fast rotation or partial cloud coverage. The theoretical models predict that these mechanisms can cause polarization of up to $0.1 \%$ in the optical (Marley \& Sengupta 2011). Indeed, linear polarization reaching $0.2 \%-1.7 \%$ in $R I$-bands was found in some early-L BDs (Tata et al. 2009). Our upper limit of $0.07 \%$ argues against the presence of any of these polarizing mechanisms in W10-53.

\section{SUMMARY AND CONCLUSIONS}

We assign spectral type L8 \pm 1 to the primary and T1.5 \pm 2 to the secondary. This classification is consistent with the NIR magnitudes of the two components, excluding the possibility that W10-53 is a nearly equal mass, hierarchical BD system.

The relations between spectral type and effective temperature $T_{\text {eff }}$ of Stephens et al. (2009) yield $T_{\text {eff }} \approx 1350$ ( \pm 60 random, \pm 100 systematic) $\mathrm{K}$ for the primary, and 1210 ( \pm 40 random, \pm 100 systematic) $\mathrm{K}$ for the secondary. The random errors reflect the template matching, and the systematic ones reflect the calibration's rms. Compared with the DUSTY (Chabrier et al. 2000; Baraffe et al. 2002) and 
BT-Settl (Allard et al. 2011) theoretical 1 Gyr isochrones, these $T_{\text {eff }}$ values correspond to masses of $0.04-0.05 M_{\odot}$ for the primary, and $0.03-0.05 M_{\odot}$ for the secondary. At 10 Gyr their masses would still be in the sub-stellar regime: $0.065-0.072 M_{\odot}$ and $0.06-0.07 M_{\odot}$, respectively. An evaluation of masses based on the NIR magnitudes alone yields similar ranges, albeit wider due to the larger uncertainties. With these masses and assuming a circular orbit with a radius equal to the projected separation at the parallax-derived distance, we obtain a period in the range of $14-20 \mathrm{yr}$, and an orbital velocity of $4.6-6.5 \mathrm{~km} \mathrm{~s}^{-1}$. Our relative radial velocity between the two components is $2.5-3.6 \mathrm{~km} \mathrm{~s}^{-1}$, implying that they are indeed bound. The actual separation between them is possibly larger than the projected one or the orbit is very eccentric.

Combining the measured radial velocities ( $V_{\mathrm{rad}}=23.1 \pm 1.1$, $19.5 \pm 1.2 \mathrm{~km} \mathrm{~s}^{-1}$ ) with the distance and the PM from Luhman (2013), following Johnson \& Soderblom (1987), we obtain Galactic velocities: $U=-17.8, V=-29.7, W=$ $-6.5 \mathrm{~km} \mathrm{~s}^{-1}$. These are not compatible with any known nearby moving group or stellar association, furthering the initial assessment of Mamajek (2013). In particular, the radial velocities we derive are too large by a factor of three to reconcile W10-53 with the 40 Myr old Argus group. This is not surprising, as Mamajek (2013) already pointed out that the NIR photometry is not compatible with such a young age. The available measurements also exclude membership into the 30 Myr old Car association (Torres et al. 2008), unless the distance to W10-53 was shortened by more than $25 \%$ (highly unlikely). A search through more than half a dozen Galactic velocity catalogs yielded a single close match - the M0 dwarf G 171-22 (Woolley et al. 1970). ${ }^{18}$ Many of the most recent PM and radial velocity catalogs do not include explicitly $U V W$, so our search is not conclusive, but it indicates that W10-53 most likely lacks nearby bright co-moving companions, and its age remains poorly constrained.

Some observations reported in this paper were obtained with the Southern African Large Telescope (SALT). All SAAO and SALT co-authors acknowledge the support from the National Research Foundation (NRF) of South Africa. This research has benefited from the MLTY dwarf compendium at http://DwarfArchives.org. We thank Adam Burgasser for making some template spectra available to us and the anonymous referee for the suggestions that helped to greatly improve the paper. R.K. and J.B. acknowledge partial support from FONDECYT through grant Nos. 1130140 and 1120601, respectively. D.M. is supported by BASAL CATA PFB-06 and FONDECYT No. 1130196.

\section{REFERENCES}

Allard, F., Guillot, T., Ludwig, H.-G., et al. 2003, in IAU Symp. 211, Brown Dwarfs, ed. C. Johns-Krull, M. K. Browning, \& A. A. West (San Francisco, CA: ASP), 325

Allard, F., Homeier, D., \& Freytag, B. 2011, in ASP Conf. Ser. 448, 16th Cambridge Workshop on Cool Stars, Stellar Systems, and the Sun, ed. C. Johns-Krull, M. K. Browning, \& A. A. West (San Francisco, CA: ASP), 91 Artigau, É., Radigan, J., Folkes, S., et al. 2010, ApJL, 718, L38

Baraffe, I., Chabrier, G., Allard, F., \& Hauschildt, P. H. 2002, A\&A, 382, 563

Barnard, E. E. 1916, AJ, 29, 191

Buckley, D. A. H., Swart, G. P., \& Meiring, J. G. 2006, Proc. SPIE, $6267,62670 \mathrm{Z}$

Burgasser, A. J., Kirkpatrick, J. D., Liebert, J., \& Burrows, A. 2003, ApJ, 594,510

Burgasser, A. J., Sheppard, S. S., \& Luhman, K. L. 2013, ApJ, submitted (arXiv:1303.7283)

Burgh, E. B., Nordsieck, K. H., Kobulnicky, H. A., et al. 2003, Proc. SPIE, 4841,1463

Chabrier, G., Baraffe, I., Allard, F., \& Hauschildt, P. 2000, ApJ, 542, 464

Crawford, S. M., Still, M., Schellart, P., et al. 2010, Proc. SPIE, 7737, 773725

Dupuy, T. J., \& Liu, M. C. 2012, ApJS, 201, 19

Eggen, O. J. 1962, RGOB, 51, 79

Epchtein, N., Deul, E., Derriere, S., et al. 1999, A\&A, 349, 236

Gandhi, P., Done, C., Ivanov, V. D., \& Huelamo, N. 2013, ATel, 5012

Gillon, M., Triaud, A. H. M. J., Jehin, E., et al. 2013, A\&A, submitted (arXiv:1304.0481)

Johnson, D. R. H., \& Soderblom, D. R. 1987, AJ, 93, 864

Kirkpatrick, J. D., Reid, I. N., Liebert, J., et al. 1999, ApJ, 519, 802

Kirkpatrick, J. D., Reid, I. N., Liebert, J., et al. 2000, AJ, 120, 447

Kniazev, A. Y., Zijlstra, A. A., Grebel, E. K., et al. 2008, MNRAS, 388, 1667

Kobulnicky, H. A., Nordsieck, K. H., Burgh, E. B., et al. 2003, Proc. SPIE, 4841,1634

Kuchinskas, A., Dobrovolskas, V., Lazauskaite, R., Lindegren, L., \& Tanabé, T. 2008, BaltA, 17, 283

Lawrence, A., Warren, S. J., Almaini, O., et al. 2007, MNRAS, 379, 1599

Lucas, P. W., Tinney, C. G., Burningham, B., et al. 2010, MNRAS, 408, L56

Luhman, K. L. 2013, ApJL, 767, L1

Mamajek, E. E. 2013, arXiv:1303.5345

Marley, M. S., \& Sengupta, S. 2011, MNRAS, 417, 2874

McLean, I. S., McGovern, M. R., Burgasser, A. J., et al. 2003, ApJ, 596, 561

Nagayama, T., Nagashima, C., Nakajima, Y., et al. 2003, Proc. SPIE, 4841, 459

O'Donoghue, D., Buckley, D. A. H., Balona, L. A., et al. 2006, MNRAS, 372, 151

Potter, S. B., Buckley, D. A. H., O’Donoghue, D., et al. 2010, MNRAS, 402,1161

Skrutskie, M., Cutri, R. M., Stiening, R., et al. 2006, AJ, 131, 1163

Stephens, D. C., Leggett, S. K., Cushing, M. C., et al. 2009, ApJ, 702, 154

Stetson, P. B. 1987, PASP, 99, 191

Tata, R., Martín, E. L., Sengupta, S., et al. 2009, A\&A, 508, 1423

Torres, C. A. O., Quast, G. R., Melo, C. H. F., \& Sterzik, M. F. 2008, in Handbook of Star Forming Regions: Vol. II, The Southern Sky, ASP Monograph, ed. B. Reipurth, 757

Woolley, R., Epps, E. A., Penston, M. J., \& Pocock, S. B. 1970, Catalogue of Stars within Twenty-five Parsecs of the Sun, Royal Observatory Annals, no. 5 (Herstmonceux: Royal Greenwich Observatory)

Wright, E. L., Eisenhardt, P. R. M., Mainzer, A. K., et al. 2010, AJ, 140, 1868

Zasov, A. V., Kniazev, A. Y., Pustilnik, S. A., et al. 2000, A\&AS, 144, 429

\footnotetext{
${ }^{18}$ Eggen (1962) lists $U=-19.6, V=-29.3, W=-6.7 \mathrm{~km} \mathrm{~s}^{-1}$ for
} HD 55383 but our re-calculation shows that the sign of $U$ is flipped. 